\title{
Multi-Metric Performance for OLSR Routing Protocol in Mobile AD-Hoc NETWORKS
}

\author{
Abdelkabir Sahnoun ${ }^{1}$, Jamal El Abbadi ${ }^{1}$ and AhmedHabbani ${ }^{2}$ \\ ${ }^{1}$ LEC laboratory, EMI,Mohammed V University, Rabat, Morocco \\ ${ }^{2}$ SIME laboratory, ENSIAS,Mohammed V University,Rabat, Morocco
}

\begin{abstract}
In the design of mobile ad hoc networks, a challenging problem is how to route information reliably and efficiently from one node to another in moderate to high level of mobility. Due to mobility, limited residual energy of the node, selection of stable and durable path for the communication is the challenge. Also remaining queuing capacity is also affected on the packet loss. Many proposals have been addressed to this problem; however, few papers consider a proactive protocol like Optimized Link State Routing Protocol $(O L S R)$ to better manage the energy consumption and link stability in routing path selection algorithms. In this paper, we explored modification to MPR selection and integrating appropriate routing metrics in the routing decision scheme to lessen effects of reason that lead to more packet loss. Our power-aware version of OLSR is proven by simulations in NS3 under a range of different mobile scenarios and network density. Significant performance gains are obtained in terms of packet loss ratio and MPR count for our modified OLSR version.
\end{abstract}

\section{KEYWORDS}

Multi-metric, Mobile Ad-hoc Network, OLSR, Energy, Link Stability

\section{INTRODUCTION}

In the last years, mobile ad hoc networks (MANETs) have attracted many research efforts [1] [2]. The challenge of scheming routing protocols for a MANET comes from two features: the mobility of the nodes and the ad hoc nature of the network, which lead to a highly dynamic topology for an infrastructure-less network. The wireless sensor networks, and also mesh networks, can be considered as a subcategory of MANETs, when the sensors or nodes are mobile. For example, a moving vehicle may be equipped with a sensor for communication purpose, or carried by a human or an animal for monitoring purpose. It is usually a less expensive and lowpowered wireless device.

There are several applications of MANET like audio, video, multimedia etc, which requires good communication and QoS [3] [4]. Similarly other wireless network CDMA, GSM and Wi-Fi, MANET is unable to provide reliable QoS [5]. Therefore, selecting appropriate protocol is important and challenging task, due to number of protocols presented in the literature, differ from each other and required guarantee of stringent QoS [6]. The main aim of QoS routing [7] is to find relevant path that must satisfy QoS constraint requirements such as, packet loss, bandwidth, delay, jitter, energy consumption which are transmission characteristics of topology. QoS routing also satisfies constraint like link, path and tree constraint [8]. Where, bandwidth, jitter-delay and end-to-end delay are main, link and path constraint respectively [9]. Thus, to satisfy the above constraints with multiple objectives, there is need of potentially new approach or technique for solving the QoS routing. To solve QoS routing, past researchers used various meta-heuristic 
algorithms [10] [11]. But, there is necessity of enhancing routing protocols in MANETS, to provide stringent QoS enhancement [12]. Many routing protocols have been proposed for MANETs. These protocols can be classified into three different groups: proactive, reactive and hybrid. In proactive routing protocols such as DSDV [13] and OLSR [14], the routes to all the destination (or parts of the network) are determined at the start up, and maintained by using a periodic route update process. In reactive protocols such as AODV [15], DSR [16], routes are determined when they are required by the source using a route discovery process. Hybrid routing protocols combines the basic properties of the first two classes of protocols into one.

In this paper we investigate an energy-aware and link stability mechanism suitable to be integrated with a proactive routing protocol. Specifically, our system is built as an energy-aware extension to OLSR [14]. The energy behavior of OLSR protocol has been evaluated and many energy-efficient designing schemes for it have been presented to optimize energy consumption, some of these approaches have based on exploring the suitability of the protocol for QoS routing [17], to achieve the best results, these QoS protocol variants typically modify both the MPR selection criteria and the path determination algorithm. While other approach have investigated a combination of multiple network parameters that indicate energy depletion and enable effective prediction of low energy paths, by identify the reasons that lead to energy depletion in different parts of network and then choose metrics to reduce their effect [18]. However, in this work we proposed a new method for determining more energetic-efficient and stable route in the network during selecting MPR nodes, via multiple metrics such as residual energy, link stability and MAC queuing capacity of the MPR node. The performance of this work has been evaluated using mobility enhancements made to NS-3 simulator in terms of packet lost ratio, end-to-end delay and number of MPRs.

The rest of the paper is organized as follows: The related work is reviewed in Section 2. In section 3 we briefly introduce OLSR protocol mechanism. We describe our multi-metric routing scheme in section 4. Simulation environment and results are presented in Section 5, and conclusion is articulated in Section 6.

\section{RELATED WORK}

MANET routing metrics in terms of energy and QoS were examined either independently or all together in some studies on OLSR. Regarding to the modified functionalities of OLSR, these investigations can be categorized into two main classes. The first class highlights the improvement of the MPR selection mechanism. However, the second class proposed modifications to link metrics for route computation. A combination of both classes is also proposed in other works. hence, in [19] and [20], both proposed to modify the MPR selection mechanism of the standard OLSR protocol by adopting the residual energy levels instead of twohop neighbors of the node. Furthermore, in [21], authors suggested a Fuzzy logic based on the MPR selection mechanism, called QMPR. It is based on the energy, stability and buffer occupancy of the nodes to select a more stable MPR and enhance energy efficiency and network lifetime. Meanwhile in [22] authors proposed modifications on OLSR by considering the delay and bandwidth as metrics for the MPR selection. However, none of the modifications mentioned above considered any energy metrics or QoS parameters for route computation. In [23], another investigation of energy efficient OLSR protocol, called OLSR_EA was offered. The authors changed the route determination algorithm to select paths based on a composite energy cost by combining the residual energy and consumed transmission power of each node. They used the auto-regressive integrated moving average time series method to measure and predict the perinterval energy consumption. A QoS version of the OLSR protocol, relying on link bandwidth as a QoS link parameter was also suggested in [24]. This QoS OLSR protocol was established to select paths with a maximum bottleneck bandwidth. In order to provide a particular path with 
International Journal of Wireless \& Mobile Networks (IJWMN) Vol. 9, No. 3, June 2017

optimal bandwidth, changes in the link bandwidth must be broadcasted. However, both approaches keep the native MPR mechanism, which is not efficient in terms of energy.

many studies have combined both MPR selection and path determination to improve the energy efficiency or QoS metrics in OLSR. For example, in [25], the authors proposed EOLSR by modifying OLSR to select MPR and find a path based on the energy. The optimal path was selected from all possible paths based on the min-max energy consumption concept. The effect of overhearing on the performance of this method was also studied. Another similar scheme was offered in [26]. The authors presented OLSRE, an energy efficient routing based on the OLSR. In this OLSRE, the energy consumption was taken into consideration during packet routing by calculating the cost of packet transmission. Moreover, different variants of MPR selection based on the node's residual energy and its number of neighbors were compared to get the best tradeoff between overhead and energy consumed. By contrary, multiple QoS metrics have been also taken in account in both MPR selection and best path selection in a few studies. The QoS extension of OLSR (QOLSR) was proposed in [27] to select MPR and find an optimal route that satisfies the end-to-end QoS requirement, often given in terms of bandwidth or delay. In [28], the authors proposed a multi-objective Algorithm based on OLSR (OLSR_MO). It was developed to fulfill three objectives: maximizing the packet delivery ratio, minimizing the average end-to-end delay, and extending the network lifetime. In order to fulfill these goals, the authors considered three routing metrics: node's queuing delay, node's energy cost, and link's stability cost. All the metrics were measured locally at each node and used to predict the corresponding future values, which are broadcasted periodically to its neighbors.

In this work, we propose a new model to select more stable, durable path with the help of residual energy, link stability and MAC queue size of the node. Certain threshold values are set to update the willingness values for MPR selection. To demonstrate our contribution, we introduced this approach in OLSR protocol, which is proactive in nature.

\section{A REVIEW ON OLSR PRotocol}

OLSR (Optimized Link State Routing), [14], is a proactive routing protocol where nodes periodically exchange topology information in order to establish a route to any destination in the network. It is an optimization of a pure link state routing protocol, based on the concept of multipoint relays (MPRs). First, using multipoint relays reduces the size of the control messages: rather than declaring all its links in the network, a node declares only the set of links with its neighbors that have selected it as "multipoint relay". The use of MPRs also minimizes flooding of control traffic (figure 1). Indeed only multipoint relays forward control messages. This technique significantly reduces the number of retransmissions of broadcast messages. OLSR consists of two main functionalities:

-Neighborhood discovery. Each node acquires the knowledge of its one-hop and two-hop neighborhood by periodic Hello messages. It independently selects its own set of multipoint relays (MPRs), among its one-hop neighbors in such a way that its MPRs cover (in terms of radio range) all its two-hop neighbors.

-Topology dissemination. Each node also maintains topological information about the network obtained by TC (Topology Control) messages, broadcast by MPR nodes.

Each node computes its routing table by the Dijkstra algorithm. This table provides the shortest route (i.e. the route with the smallest hop number) to any destination in the network. 


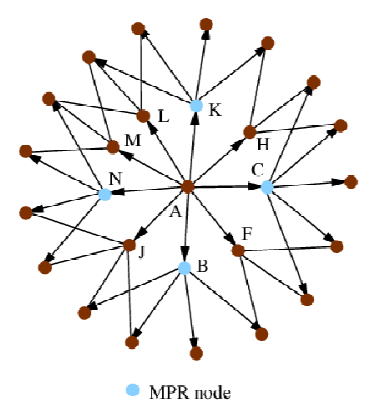

Figure 1. MPR Mechanism

\section{Proposed Modeling System}

To improve the QoS of mobile ad hoc network, we have proposed a new module we designed and implemented in the OLSR, which is based on the residual energy, link stability and queuing capacity of the node. In native OLSR, selection of MPRs is based on the willingness and degree of reachability of the nodes. In our proposed approach, we made essentially two modifications to OLSR, first we take into account a combination of multiple network parameters that indicate link stability, energy depletion and MAC queue utilization to select more stable and durable path. Then we added modifications to the MPR selection criteria.

Since the dynamic topology changes frequently in MANETs, the reliability of a path depends on the stability of each link of this path, we adopt the duration of time between two neighbors in order to estimate that two neighbors remain connected or not by using the motion parameters such as speed, direction and distance. The measure used in this research to represent the time for which two mobile nodes can remain in contact with each other is the link expiration time (LET). To find the estimated LET in our proposed routing metric, we used the following formula as given in [29]. Let $\mathrm{i}$ and $\mathrm{j}$ be two mobile nodes within the transmission range $r$ of each other, let $\left(\mathrm{x}_{\mathrm{i}}, \mathrm{y}_{\mathrm{i}}\right)$ and $\left(x_{j}, y_{j}\right)$ be the coordinate of $i$ and $j$. Also let $\left(v_{i}, \theta_{i}\right)$ be the speed and the moving direction of the node $i$, and $\left(v_{j}, \theta_{j}\right)$ be the speed and the moving direction of the node $j$ (figure 2 ). then the amount of time two mobile nodes $i$ and $j$ will stay connected LET $(i, j)$ is predicted by:

$\operatorname{LET}(i, j)=\frac{-(a b+c d)+\sqrt{\left(a^{2}+c^{2}\right) r^{2}-(a d-b c)^{2}}}{a^{2}+c^{2}}$

Where

$$
\begin{array}{ll}
a=v_{i} \cos \theta_{i}-v_{j} \cos \theta_{j}, & b=x_{i}-x_{j}, \\
c=v_{i} \sin \theta_{i}-v_{j} \sin \theta_{j}, & d=y_{i}-y_{j} .
\end{array}
$$

This information can be obtained if the mobile nodes are equipped with a GPS system. Note that when $v_{i}=v_{j}$ and $\theta_{i}=\theta_{j}$, LET $(i, j)$ becomes $\infty$. 


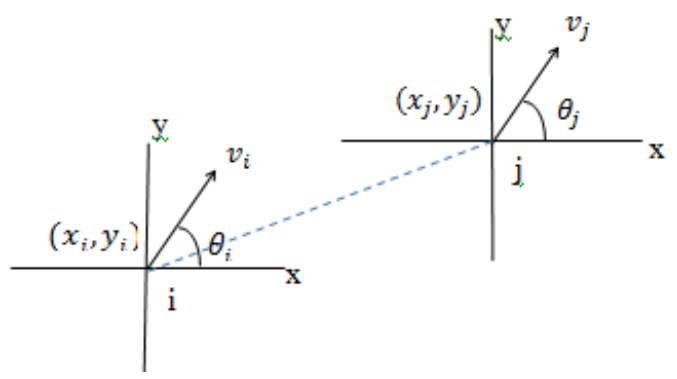

Figure 2. Link Expiration Time Architecture

When a source node sends a request packet, the packet appends its location, direction and speed. The next hop neighbor of the source node that receives the request packet will be able to compute the duration of time between itself and the source node.

Assume that $\mathrm{P}$ is a routing path between source and destination, and $\left(\mathrm{P}_{1}, \mathrm{P}_{2}, \ldots, \mathrm{P}_{\mathrm{k}}\right)$ is the set of all the links along $\mathrm{P}$. The path stability $\mathrm{S}(\mathrm{P})$ is the minimum of the $\operatorname{LET}\left(\mathrm{P}_{\mathrm{i}}\right)$ along the path $(\mathrm{i} \in$ $(1,2, \ldots, \mathrm{k}))$

$$
S(P)=\min _{i \epsilon(1,2, \ldots, k)}\left(\operatorname{LET}\left(P_{i}\right)\right)
$$

With regard to energy aware, for increasing the network lifetime without loss of performance, we take into account cross layer parameters which contain residual energy of nodes and network congestion parameters, and then modify OLSR in order to make routing decisions according to these parameters. We combine these metrics to compute an energetic cost for each node i, as shown in Equation 3.

$$
E C_{i}=\frac{N P_{i}}{M Q_{\max }}+\left(1-\frac{R E_{i}}{I E_{\max }}\right)
$$

Where $N P_{i}$ is the number of packets in the MAC queue and $R E_{i}$ is the residual energy at each time. $M Q_{\max }$ is the maximum considered MAC queue size and $I E_{\max }$ is the initial energy of a node.

The path energetic cost $\mathrm{EC}(\mathrm{P})$ is the sum of $\mathrm{EC}_{\mathrm{i}}$ along the path $(\mathrm{i} \in(1,2, \ldots, \mathrm{k}))$,

$$
E C(P)=\sum_{i=1}^{k} \frac{N P_{i}}{M Q_{\max }}+\left(1-\frac{R E_{i}}{I E_{\max }}\right)
$$

We intended to select a more stable path with lower consumption energy cost to achieve reduction in packet lost and prolonged lifetime of the network along with QoS support. The stability S(P) of the path is obtained from Equation (2) and lower cost based on Equation (4), to get the best possible path with higher link stability and lower energetic cost, we represented the path cost by divide the energetic cost of the path $\mathrm{EC}(\mathrm{P})$ by the path stability $\mathrm{S}(\mathrm{P})$, when as the $\mathrm{EC}(\mathrm{P})$ decreases and the $\mathrm{S}(\mathrm{P})$ increases, the path cost cost $(\mathrm{P})$ will increases. The path that minimizes the cost value $\operatorname{cost}(\mathrm{P})$ is preferred.

$$
\operatorname{cost}(P)=\frac{\sum_{i=1}^{k} \frac{N P_{i}}{M Q_{\max }}+\left(1-\frac{R E_{i}}{I E_{\max }}\right)}{\min _{i \epsilon(1,2, \ldots, k)}\left(\operatorname{LET}\left(P_{i}\right)\right)}
$$


In order to not increasing network overhead, we embed links costs to the TC packet that are periodically generated by each node. So TC packet is extended to include the field for the updated costs, which is locally computed using Equations (1) and (3) of the originator node. And the Topology tuples are also extended to take a new field for the cost of the originator node. In the last step, based on the path costs computed from the links costs, routing tables should be updated rather than on number of hops. And should also include path costs to the destination address instead of the number of hops, where path cost is define as shown in Equation (5), hence a new algorithm have been proposed that assigns cost equal to 1 to paths towards the 1-hope neighbors. Next is examines the topology tuples given by the topology table and three cases are considered to update the routing table. The first one occurs when there is an entry in the routing table for the originator node of the topology tuple. In this case, a new entry is added to the routing table for the destination node of TC with cost equal to the sum of the cost corresponding to route to the originator node and the link cost from the originator node to the final node. The second case, occurs when there are entries for both the originator and the destination node of the topology tuple. Then, the algorithm chooses greedily the new path detected through the originator node or it maintains the old path, by comparing their costs. Finally, in the case where there aren't entries neither for the originator node nor the destination node, no new entry is created.

at MPR selection level, according to OLSR standard [14] each node has a parameter called 'willingness', indicates its availability to carry traffic on behalf of other nodes, the value of a node's willingness parameter is an integer between 0 and 7 , A node with willingness equal to 0 must never be selected as MPR by any node. A node with willingness equal to 7 must always be selected as MPR. But by default in OLSR standard all willingness values are set to a default value equal to 3, and it is still constant along the simulation. We put in the willingness variable the available residual energy of the node which be taken each time the HELLO packet is generated, using the Equation 6.

$$
\text { Willingness }=\text { round }\left(\frac{E_{i}}{E_{\max }} * 7\right)
$$

Where $E_{i}$ is the residual energy at each time and $E_{\max }$ is the initial energy of the node.

\section{Simulation RESUlts AND ANALYSIS}

As mentioned before we used NS3 network simulator to evaluate our modified version of OLSR. We executed the simulations to evaluate the performance of our modified routing scheme compared to the standard OLSR. We considered three performance metrics to evaluate this proposition, which are:

- Packet Loss Ratio: This metric represents the total number of lost packets in the network. This metric is most important QoS metric which demonstrates the affect of durable and stable approach on total number of lost packets in the network.

- MPR Count: This metric represents the number of different MPR nodes in the network.

- End-to-end delay: Time required for the successful transmission of the packet is taken as end-to-end delay.

We simulated a MANET with varied number of nodes in a dense $1000 \times 1000$ meter square area. There are 5 UDP sources generating packets of 512 bytes with different data rates. We have two variations of the simulation setup to evaluate the performance of our modified routing scheme compared to the standard OLSR. The common simulation parameters of the two variations are summarized in Table 1 below. 
Table 1. Simulation parameters

\begin{tabular}{|l|l|}
\hline Area & $1000 \times 1000$ \\
\hline Mobility Model & Random Waypoint Mobility model \\
\hline Traffic sources & 5 \\
\hline Traffic Type & CBR / UDP \\
\hline Packet Size & $512 \mathrm{bytes}$ \\
\hline Start of Traffic & $50 \mathrm{sec}$ \\
\hline Transmission Power & $7.5 \mathrm{dbm}$ \\
\hline Link bandwidth & $2 \mathrm{Mb} / \mathrm{s}$ \\
\hline Simulation Time & $300 \mathrm{sec}$ \\
\hline
\end{tabular}

In the first setup, we consider a mobile scenario where mobile nodes move in the area based on a Random Waypoint Mobility model with a speed varied from 10 to $50 \mathrm{~m} / \mathrm{s}$, and the number of nodes are varied from 20 to 50, with the parameters described in Table 1.

From Fig 3, we show the performance of the standard OLSR and the modified OLSR over packet loss ratio metric. As the total number of nodes in the network increases, the packet loss ratio decreases in the network. From this figure it's obvious that the modified OLSR scheme performs better than standard OLSR. As number of nodes increases chances of selecting better MPR from neighbor nodes are increased. By selecting more stable and durable MPR nodes reduces the chances of path break as compared to other approaches and also the chances of queue overflow are reduced in our modified OLSR protocol, which results in to minimizing packet loss in the network.

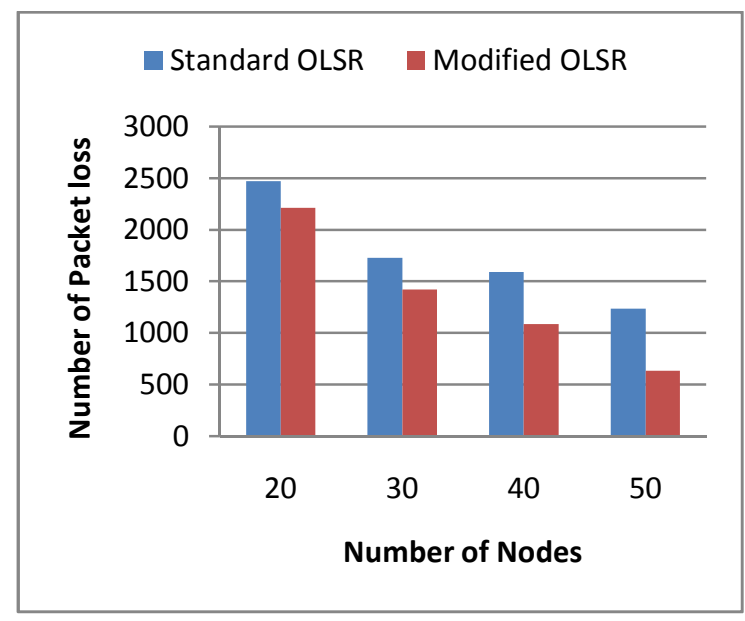

Figure 3.Packet Loss Vs Number of Nodes

Fig 4 shows the performance of the standard OLSR and the modified OLSR protocols over the MPR count. The number of the MPRs in the network increases and after certain number of nodes, MPR count stabilizes. By varying nodes, it is observed that, the MPR nodes in the network are minimized as compared to the standard OLSR. The number of MPR nodes in the network is minimized in modified OLSR as compare to the standard because it elects the durable MPR nodes in the network which results in the stable and durable topology. 
International Journal of Wireless \& Mobile Networks (IJWMN) Vol. 9, No. 3, June 2017

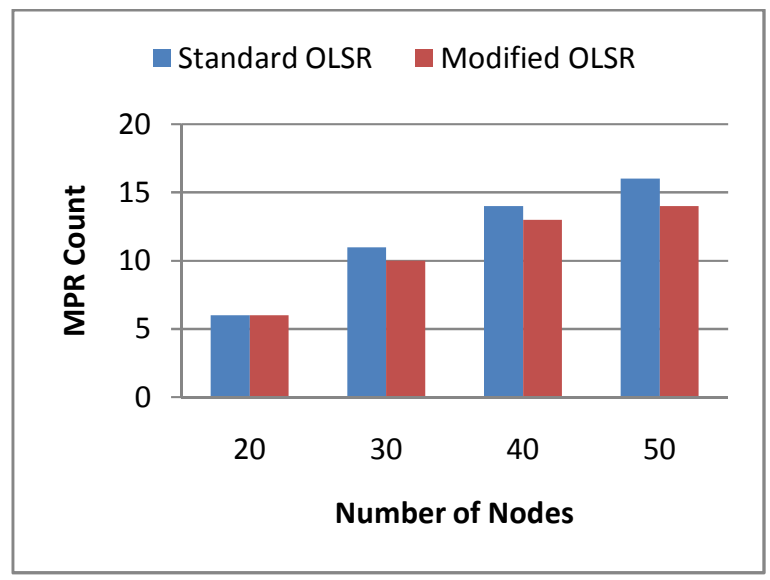

Figure 4. MPR Count Vs Number of Nodes

Fig 5 shows the performance of the standard OLSR and the modified one with the help of end-toend delay metric. As the number of nodes in the network increases, end-to-end delay is slightly increased in modified OLSR protocol, this is due to at every node computation is more complex than standard OLSR protocol.

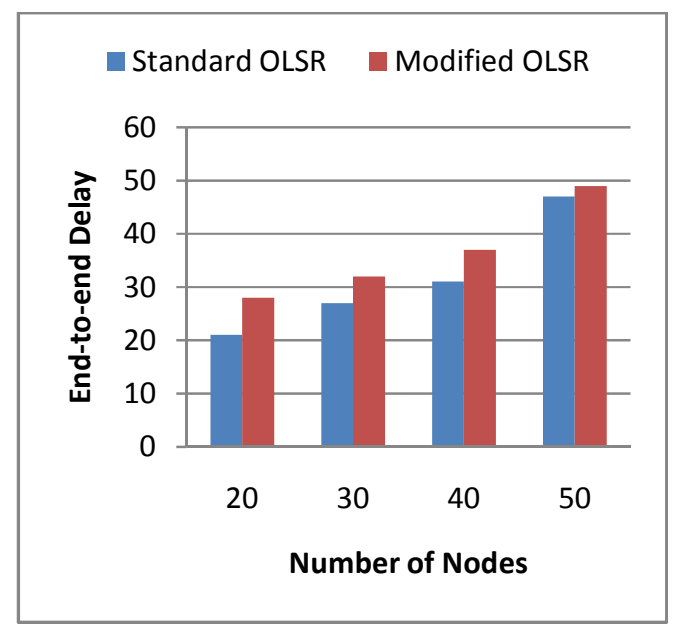

Figure 5.End-to-end Delay Vs Number of Nodes

In the second setup, to evaluate the performance of our modified OLSR, speed of the node is varied by keeping the other parameters fixed during the simulation.

Fig 6 shows the impact of speed of nodes on packet loss in the network in OLSR and modified OLSR protocols. As speed increases the more number of packets are lost in the network. This happens due to, as speed increases, there are more chances of path break, which results into the loss of packets which are at intermediate nodes, links of the route. Our modified OLSR reduces the packet loss in the network which guarantees the QoS of the network. 
International Journal of Wireless \& Mobile Networks (IJWMN) Vol. 9, No. 3, June 2017

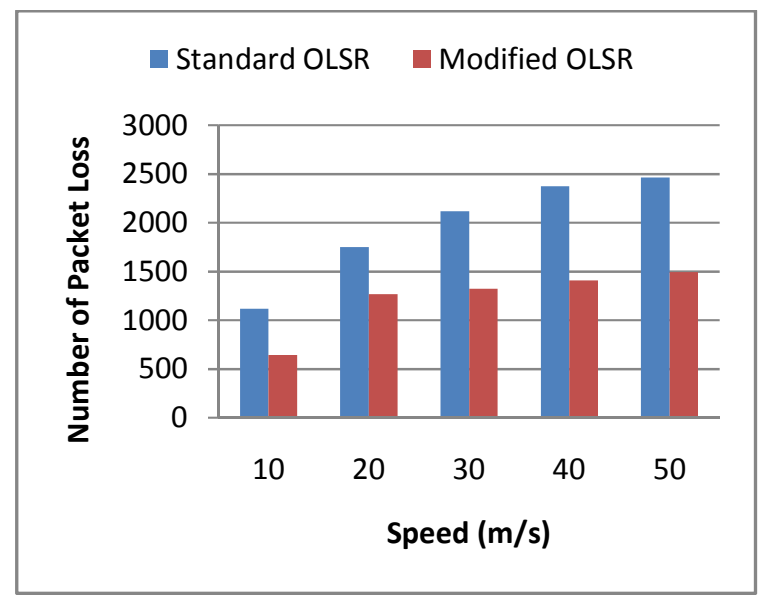

Figure 6. Number of Packet Loss Vs Speed

Fig 7 shows the impact of speed on number of MPR nodes in the network, MPR count goes on increasing linearly with increase in node speed. As speed of the nodes increases, the frequency of path break increased which results into increase in MPR count. The modified OLSR reduces the number of MPR counts compared to the standard one.

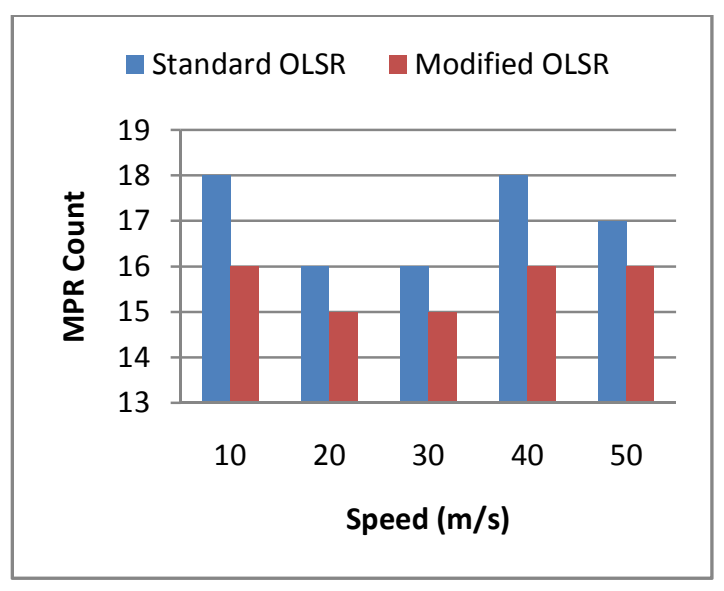

Figure 7. MPR Count Vs Speed

Fig 8 shows the impact of speed on the end-to-end delay. Delay also goes on increasing when speed of nodes increased. This is due to when packet is in network and route is unavailable (due to path break), requires time to recalculate route. As speed increases, frequency of path break is more. The modified OLSR minimized the end-to-end delay as compared to standard OLSR at higher speed. Although, requires more time for computation, but able to select durable and stable path which reduces path break frequency, results into reduction in delay. 


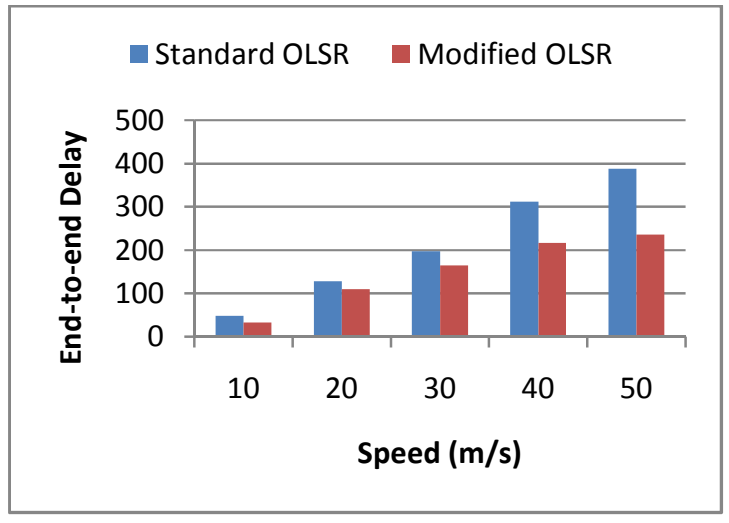

Figure 8. End-to-end Delay Vs Speed

\section{Conclusions}

In this paper we demonstrated an effective and efficient energy-aware and link stability approach for the proactive MANET routing protocol OLSR, by a mechanism that aim to increase network lifetime and mitigate end-to-end delay and packet loss ratio, we are interested in integrating appropriate routing metrics in the routing decision scheme to reduce effects of reason that lead to more energy consumption, via adopting three salient parameters which are: residual energy, link stability and network topology. Then we proposed a novel MPR selection policy that allows network lifetime to be preserved for longer time, by involve the residual energy in MPR selection criteria through the willingness variable. We evaluated the modified OLSR under a range of different scenarios, varying number of nodes and mobility pattern, we compared our modified OLSR, in terms of packet loss ratio, MPR count and end-to-end delay, with the standard OLSR. Our simulation showed that our modified OLSR is able to enhance end-to-en delay and packet loss ratio pretty more than precedent work without significant loss in terms of end-to-end delay. We are currently extending our OLSR modifications to investigate more cross layer parameters that allow increasing the network lifetime.

\section{REFERENCES}

[1] C. S. R. Murthy, B. Manoj, Ad Hoc Wireless Networks: Architectures and Protocols, Portable Documents, Pearson education, 2004.

[2] I. Chlamtac, M. Conti, J. J.-N. Liu, Mobile ad hoc networking: imperatives and challenges, Ad hoc networks 1 (1) (2003) 13-64.

[3] W. Castellanos, J. C. Guerri, P. Arce, Performance evaluation of scalable video streaming in mobile ad hoc networks, IEEE Latin America Transactions 14 (1) (2016) 122-129.

[4] R. A. Gu'erin, A. Orda, Qos routing in networks with inaccurate information: theory and algorithms, IEEE/ACM Transactions on Networking (TON) 7 (3) (1999) 350-364.

[5] F. Kuipers, P. Van Mieghem, T. Korkmaz, M. Krunz, An overview of constraint-based path selection algorithms for qos routing, IEEE Communications Magazine, 40 (12)(2002).

[6] D. H. Lorenz, A. Orda, Qos routing in networks with uncertain parameters, Networking, IEEE/ACM Transactions on 6 (6) (1998) 768-778.

[7] X. Hannan, C. K. Chaing, S. K. G. Winston, Quality of service models for ad hoc wireless networks, in: The handbook of ad hoc wireless networks, CRC Press, Inc., 2003, pp. 467-482.

[8] P. Basarkod, S. Manvi, Mobility and qos aware anycast routing in mobile ad hoc networks, Computers \& Electrical Engineering 48 (2015) 86-99.

[9] Z. Wang, Y. Chen, C. Li, Corman: A novel cooperative opportunistic routing scheme in mobile ad hoc networks, IEEE Journal on Selected Areas in Communications 30 (2) (2012) 289-296.

[10] L. Zhang, L.-b.Cai, M. Li, F.-h. Wang, A method for least-cost qos multicast routing based on genetic simulated annealing algorithm, Computer Communications 32 (1) (2009) 105-110. 
[11] X. Yuan, X. Liu, Heuristic algorithms for multi-constrained quality of service routing, in: INFOCOM 2001. Twentieth Annual Joint Conference of the IEEE Computer and Communications Societies.Proceedings. IEEE, Vol. 2, IEEE, 2001, pp. 844-853.

[12] M. Abolhasan, T. Wysocki, E. Dutkiewicz, A review of routing protocols for mobile ad hoc networks, Ad hoc networks 2 (1) (2004) 1-22.

[13] C. E. Perkins and P. Bhagwat, " Highly dynamic Destination-Sequenced Distance-Vector routing (DSDV) for mobile computers", In Proceedings of the SIGCOMM '94 Conference on Communications Architectures, Protocols and Applications, 1994, pp. 234-244.

[14] T. Clausen and P. Jacquet "Optimized Link State Routing Protocol (OLSR)." RFC 3626, IETF Network Working Group, October 2003.

[15] C. E. Perkins and E. M. Royer, “Ad Hoc On-demand Distance Vector Routing," In Proceedings of the 2nd IEEE Workshop on Mobile Computing Systems and Applications, 1999, pp. 90-100.

[16] D. B. Johnson and D. A. Maltz, "Dynamic Source Routing in Ad-Hoc Ad hoc Networks", Mobile Computing, 1996, pp. 153-181.

[17] K. Kowalik et al., "Making OLSR Aware of Resources", Proc. Int. Conf. on Wireless Communications, Networking and Mobile Computing, 2007, Sept. 2007, pp. 1488-1493.

[18] E. Paraskevas, K. Manousakis, S. Das and John S. Baras, "Multi-Metric Energy Efficient Routing in Mobile Ad-Hoc Networks", 2014 IEEE Military Communications Conference.

[19] De Rango, F., Fotino, M., \&Marano, S. (2008). EE-OLSR: Energy efficient OLSR routing protocol for mobile ad-hoc networks. (pp. 1-7). IEEE.

[20] Ghanem, N., Boumerdassi, S., \& Renault, E’. (2005). New energy saving mechanisms for mobile adhoc networks using OLSR.In Proceedings of the 2nd ACM international workshop on Performance evaluation of wireless ad hoc, sensor, and ubiquitous networks, (pp. 273-274).ACM.

[21] Kots, A., \& Kumar, M. (2014). The fuzzy based QMPR selection for OLSR routing protocol.Wireless Networks, 20(1), 1-10.

[22] Munaretto, A., \& Fonseca, M. (2007). Routing and quality of service support for mobile ad hoc networks.Computer Networks, 51(11), 3142-3156.

[23] Guo, Z., Malakooti, S., Sheikh, S., Al-Najjar, C., Lehman, M., \&Malakooti, B. (2011). Energy aware proactive optimized link state routing in mobile ad-hoc networks. Applied Mathematical Modelling, 35(10), 4715-4729.

[24] Ge, Y., \& Kunz, T. (2003).Lamont, L. Proactive QoS routing in ad hoc networks: Springer.

[25] Benslimane, A., El Khoury, R., El Azouzi, R., \& Pierre, S. (2006). Energy power-aware routing in OLSR protocol.In Mobile Computing and Wireless Communication International Conference, 2006.MCWC 2006. Proceedings of the First(pp.14-19). IEEE.

[26] Mahfoudh, S., \&Minet, P. (2008). An energy efficient routing based on OLSR in wireless ad hoc and sensor networks. In22nd International Conference on Advanced Information Networking and Applications-Workshops, 2008.AINAW 2008 (pp.1253-1259).IEEE.

[27] Badis, H., \& Al Agha, K. (2005). QOLSR, QoS routing for ad hoc wireless networks using OLSR. European Transactions on Telecommunications, 16(5), 427-442.

[28] Guo, Z., Malakooti, S., Sheikh, S., Al-Najjar, C., \&Malakooti, B. (2011). Multi-objective OLSR for proactive routing in MANET with delay, energy, and link lifetime predictions.Applied Mathematical Modelling, 35(3), 1413-1426.

[29] Su, W., Lee, S.-J., Gerla, M.: Mobility prediction in wireless networks. In: Proceedings of the IEEE Military Commu., Conference (MILCOM), Los Angeles, October 2000, pp. 91- 195 (2000) 